\title{
Recent advances in the imaging of hepatocellular carcinoma
}

\author{
Myung-Won You ${ }^{1,2,}$, So Yeon Kim, ${ }^{1,2}$ Kyoung Won Kim, ${ }^{1,2}$, So Jung Lee ${ }^{1,2}$, Yong Moon Shin ${ }^{1,2}$, Jin Hee Kim1, and Moon-Gyu Lee ${ }^{1,2}$ \\ 'Department of Radiology and Research Institute of Radiology, ${ }^{2}$ Asan Liver Cancer Center, Asan Medical Center, University of Ulsan \\ College of Medicine, Seoul; ${ }^{3}$ Department of Radiology, Eulji Hospital, Eulji University School of Medicine, Seoul, Korea
}

The role of imaging is crucial for the surveillance, diagnosis, staging and treatment monitoring of hepatocellular carcinoma (HCC). Over the past few years, considerable technical advances were made in imaging of HCCs. New imaging technology, however, has introduced new challenges in our clinical practice. In this article, the current status of clinical imaging techniques for $\mathrm{HCC}$ is addressed. The diagnostic performance of imaging techniques in the context of recent clinical guidelines is also presented. (Clin Mol Hepatol 2015;21:95-103)

Keywords: Hepatocellular carcinoma; Diagnostic imaging; Ultrasonography; Computed tomography; Magnetic resonance imaging

\section{INTRODUCTION}

Hepatocellular carcinoma (HCC) is the most common primary hepatic malignancy. The overall incidence of HCC is steadily rising across the world: its overall incidence remains alarmingly high in the developing countries and is increasing in most of the developed countries as well. ${ }^{2}$ Early diagnosis and accurate assessment of disease extent are crucial for appropriate clinical management of patients with HCC to help support curative treatment and excellent prognosis. ${ }^{3}$ Of various clinical diagnostic tools, in particular, imaging plays a vital role in surveillance, characterization, staging, and treatment monitoring of HCC in recent clinical guidelines proposed by the Barcelona European Association for the Study of the Liver (EASL) Conference, the Asian Pacific Association for the Study of the Liver (APASL), and the American Association for Study of Liver Diseases (AASLD) (Table 1). ${ }^{4-6}$
It is widely accepted that hepatocarcinogenesis is a multistep process. ${ }^{7}$ The two key events of the process developing simultaneously are: (1) progressive cellular morphologic and functional changes and (2) sequential changes in the intranodular blood supply including progressive decrease of the portal supply and increase in the number of unpaired arteries. ${ }^{8-10}$

These carcinogenetic changes can be detected and characterized by means of various clinical imaging techniques. For instance, cellular morphologic changes can be depicted using gray scale ultrasonography (US), unenhanced computed tomography (CT), and unenhanced magnetic resonance imaging (MRI) including diffusion-weighted imaging (DWI). On the other hand, the application of contrast agents facilitates the evaluation of functional changes held in a nodule during hepatic carcinogenesis beyond morphologic changes. Contrast-enhanced US using a new US contrast agent, Sonazoid (GE healthcare), ${ }^{11}$ and enhanced MRI with super-

\section{Abbreviations:}

AASLD, american association for study of liver diseases; ADC, apparentdiffusion coefficient; APASL, asian pacific association for the study of the liver; $\mathrm{CT}$, computed tomography; DWI, diffusion-weighted imaging; EASL, european Association for the study of the Liver; HCC, hepatocellular carcinoma; LI-RADS, liver imaging-reporting and data system; MRI, magnetic resonance imaging; OATP, organic anion transporting polypeptides; SPIO, super-paramagnetic iron oxide; US, ultrasonography
}

\section{Corresponding author : So Yeon Kim}

Department of Radiology and Research Institute of Radiology, Asan Medical Center, University of Ulsan College of Medicine, 88 Olympic-ro 43-gil, Songpa-gu, Seoul 138-736, Korea

Tel: +82-2-3010-5980, Fax: +82-2-476-4719

E-mail: sykimrad@amc.seoul.kr 
Table 1. Diagnostic Criteria of HCC

\begin{tabular}{|c|c|}
\hline AASLD $^{*} 2005$ & $\begin{array}{l}\text { Liver nodule }>10 \text { mm detected on surveillance US } \\
\text { Arterial hypervascularity and venous or delayed phase washout on single dynamic technique (4-phase MDCT/dynamic } \\
\text { contrast enhanced MRI) }\end{array}$ \\
\hline AASLD $^{*} 2010$ & $\begin{array}{l}\text { Nodule in cirrhotic liver, detected on surveillance US: } \\
\cdot \text { 10-20 mm nodule with typical vascular pattern (arterial hyperenhancement and delayed washout) on two dynamic } \\
\text { imaging studies } \\
\cdot>20 \mathrm{~mm} \text { nodule with typical vascular pattern on one dynamic imaging technique or AFP }>200 \mathrm{ng} / \mathrm{ml}\end{array}$ \\
\hline $\operatorname{APASL}^{\dagger} 2010$ & Typical vascular pattern (i.e. arterial enhancement with portal venous washout) on dynamic CT, MRI or CEUS regardless of size \\
\hline EASL $^{\ddagger} 2012$ & $\begin{array}{l}\geq 1 \text { cm nodule detected on surveillance US: } \\
\quad \text { 10-20 mm nodule; } 1 \text { or } 2 \text { positive techniques ( } 4 \text {-phase CT/ dynamic contrast enhanced MRI) with HCC radiological } \\
\quad \text { hallmarks (arterial hyperenhancement and portal or delayed washout) } \\
\quad \text { > } 20 \text { mm nodule; } 1 \text { positive technique ( } 4 \text {-phase CT or dynamic contrast enhanced MRI) with HCC radiological hallmarks }\end{array}$ \\
\hline
\end{tabular}

${ }^{\star}$ American Association for Study of Liver Diseases, ${ }^{\dagger}$ Asian Pacific Association for the Study of the Liver, ${ }^{\ddagger}$ European Association for the Study of the liver.
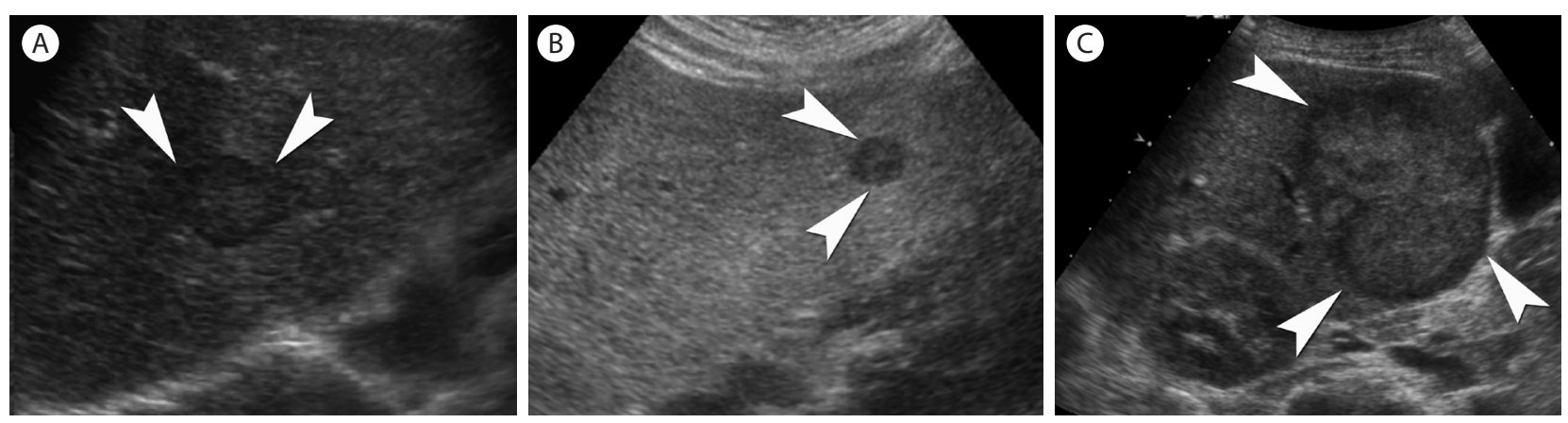

Figure 1. Various gray scale US features of HCCS. (A-C) On gray scale US, HCC (arrowheads) can be seen as a nodule with thin hypoechoic peripheral zone (A), a discrete hypoechoic nodule (B), or a mass with heterogenous echogenicity (C) in comparison to the surrounding hepatic parenchyma.

paramagnetic iron oxide (SPIO) can depict the presence of Kupffer cells in a nodule. ${ }^{12}$ Changes in signal intensity on hepatobiliary images of Gd-EOB-DTPA-enhanced MRI correlate with organic anion transporting polypeptides 8 (OATP 8) expression. ${ }^{13}$ Multiphase CT and MRI with contrast agents provide the information related to hemodynamic changes in a nodule that are currently regarded as the most important diagnostic criteria for HCC in routine clinical practice. ${ }^{4-6}$

Over the past few years, considerable technical advances were made in imaging of HCCs. New imaging technology, however, has introduced new challenges in our clinical practice. We must decide how best to standardize protocols, appropriate imaging protocols for clinical indications, and ensure diagnostic efficacy. In this article, the current status of clinical imaging techniques for HCC is addressed. The diagnostic performance of imaging techniques in the context of recent clinical guidelines is also presented.

\section{Imaging characteristics and diagnosis of HCC}

Gray scale US is the most commonly used imaging test for surveillance since it is relatively inexpensive, noninvasive, and well accepted by patients. ${ }^{4-6}$ A systematic review of 14 US studies on the accuracy of US in diagnosing HCC published the sensitivity of $69 \%$ and specificity of $97 \% .^{14}$ However, the diagnostic performance of US is significantly affected by multiple factors including the expertise of sonographer, the body habitus of patients, and size and location of lesions. ${ }^{15}$ US features of HCC are variable and may be indistinguishable from benign nodules. ${ }^{16} \mathrm{HCC}$ usually presents as a discrete nodule with heterogenous echogenicity or a mosaic pattern, and thin hypoechoic peripheral zone which represents the tumor capsule (Fig. 1). 17,18 In particular, since gray scale US reflects only nonspecific cellular morphologic changes in a nodule, its use is indicated only for the detection of $\mathrm{HCC}^{4-6}$ With US contrast agents, US can provide vascular and functional information. US contrast agents are coated microbubbles that serve as an acoustic reflector and appear as high echogenecity in the location where contrast agents present. ${ }^{19}$ Blood-pool US contrast agent such as SonoVue (Bracco Diagnostics) provides detailed real-time hemodynamic information. ${ }^{19,20}$ However, some recent data demonstrated intrahepatic cholangiocarcionoma could present with vascular patterns similar to HCC. This led a recent revision in 

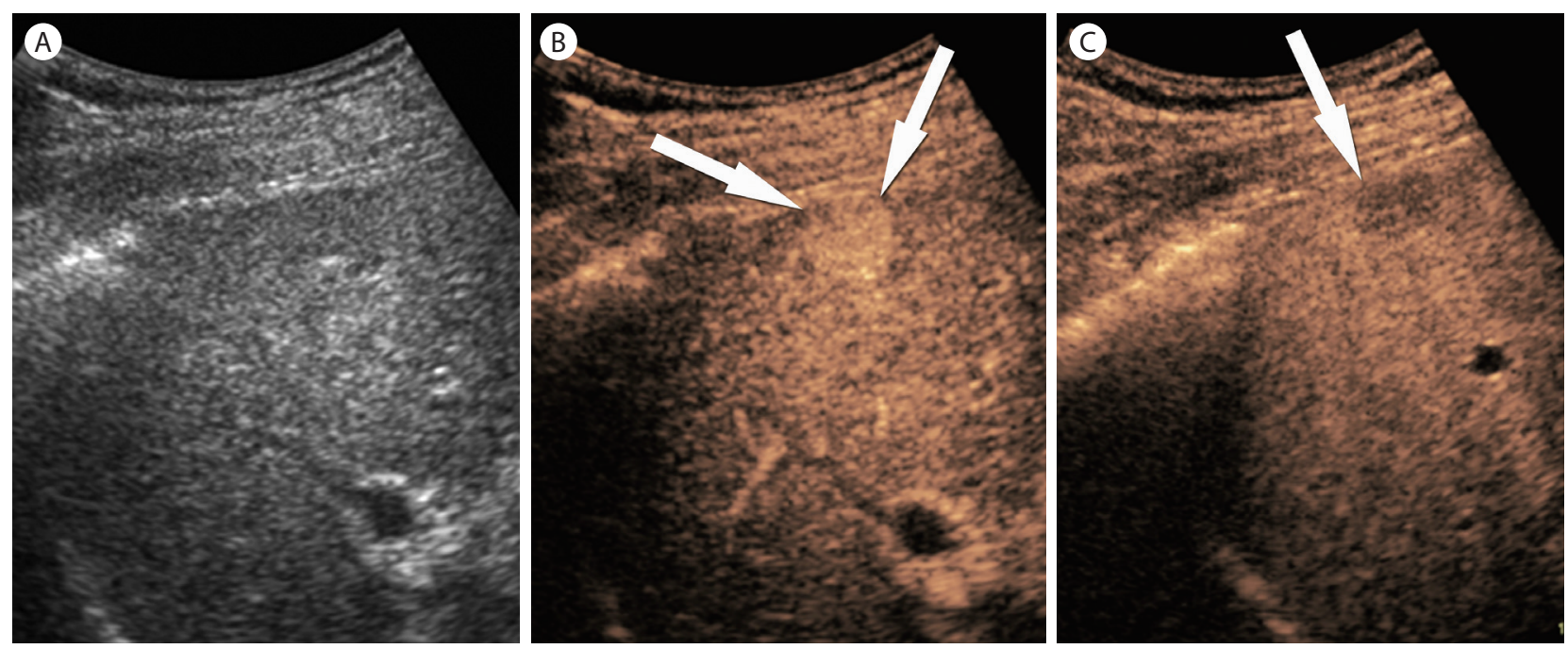

Figure 2. Contrast-enhanced US images of HCC. (A) On a gray scale US image, HCC is barely visible. (B) An arterial phase image of Sonazoid-enhanced US detects the strong arterial hypervascularity of HCC (arrows). (C) On a Kupffer phase image of Sonazoid-enhanced US, HCC (arrow) appears hypoechoic compared to the enhanced surrounding hepatic parenchyma. Thus, contrast-enhanced US using Sonazoid presents both vascular and functional information of lesions. In addition, it can possibly enhance the visibility of HCCs.

the AASLD guideline to exclude contrast-enhanced US from the list of diagnostic techniques that can be used to characterize lesions suspicious for HCC. ${ }^{21,22}$ A recently introduced US contrast agent, Sonazoid (GE healthcare) is phagocytosed by Kupffer cells of the liver that can be seen during the postvascular phase or Kupffer phase (starting $10 \mathrm{~min}$ after the injection) (Fig. 2)." This contrast agent also provides real-time hemodynamic information as a blood-pool US contrast agent in earlier phase of imaging. The enhancement characteristics of Sonazoid would help expand the role of contrast-enhanced US imaging for the surveillance and diagnosis of $\mathrm{HCC}{ }^{23,24}$ Accordingly, the APASL guideline included the indication of Sonazoid US imaging for further evaluation of lesions with atypical imaging features. ${ }^{5}$

In a common clinical setting, a hepatic nodule detected on surveillance US is further interrogated with contrast-enhanced multiphase $\mathrm{CT}$ or MRI to demonstrate the presence of a specific vascular profile (i.e., contrast wash-in during the arterial phase followed by contrast wash-out during the portal or the delayed phase) (Fig. 3)., According to the revised AASLD guidelines, the sensitivity of detecting $1-2 \mathrm{~cm} \mathrm{HCC}$ was $44 \%$ with CT and $44 \%$ with MRI, while the specificity was $100 \%$ with either CT or MRI. ${ }^{25}$ The typical vascular pattern of HCC was identified in $65 \%$ of nodules by a single technique while maintaining the high specificity. ${ }^{25}$ Another study, in support of the revised AASLD guidelines, reported that a sequential combination of the two imaging methods, requiring only one to be positive, yielded the sensitivities of $74-89 \%$ and the specificities of $91-99 \% .{ }^{26}$ Currently, CT is the most commonly used imaging method for the diagnosis and staging of HCC, because of its wide availability and high temporal and spatial resolution. On the other hand, the strengths of MRI include superb soft-tissue contrast and information on the tissue composition (Fig. 4). According to a meta-analysis, the pooled sensitivity and specificity for the detection of HCC with contrast-enhanced MRI were $81 \%$ and $85 \%$, respectively, while those with contrast-enhanced CT were $68 \%$ and $93 \%$, respectively. ${ }^{14}$ Several studies compared contrast-enhanced MRI and CT in the same patient population for the detection of HCC and published that the sensitivities were higher with MRI (61-90\%) than with CT (54-78\%). ${ }^{27-30}$ The differences in the sensitivities between the two imaging modalities were more pronounced in the detection of small HCC nodules of $1-2 \mathrm{~cm}$ in diameters (84-85\% with MRI vs. $47-68 \%$ with CT). ${ }^{27,29}$

Early HCCs frequently present with atypical contrast enhancement patterns reflecting their immature intranodular vascular changes in histological level. ${ }^{731,32}$ As many as $87 \%$ of well-differentiated lesions and $41-62 \%$ of lesions smaller than $2 \mathrm{~cm}$ may show atypical vascular patterns (Fig. 4). ${ }^{31,32}$ An important clinical quandary is that these atypical lesions are the main target of the surveillance program because they respond more likely to curative treatments than other typical late-stage lesions. ${ }^{33,34}$ However, on the basis of the AASLD guidelines that stipulate typical contrast enhancement profiles for the diagnosis of HCC, the sensitivity for detecting early HCCs is rather limited. The APASL guidelines, underscoring the usefulness of Kupffer cell specific imaging such as SPIO-enhanced MRI and Sonazoid-enhanced US, may be more 


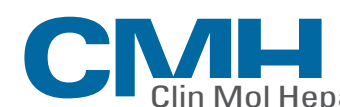

Clin Mol Hepatol

Volume_21 Number_1 March 2015
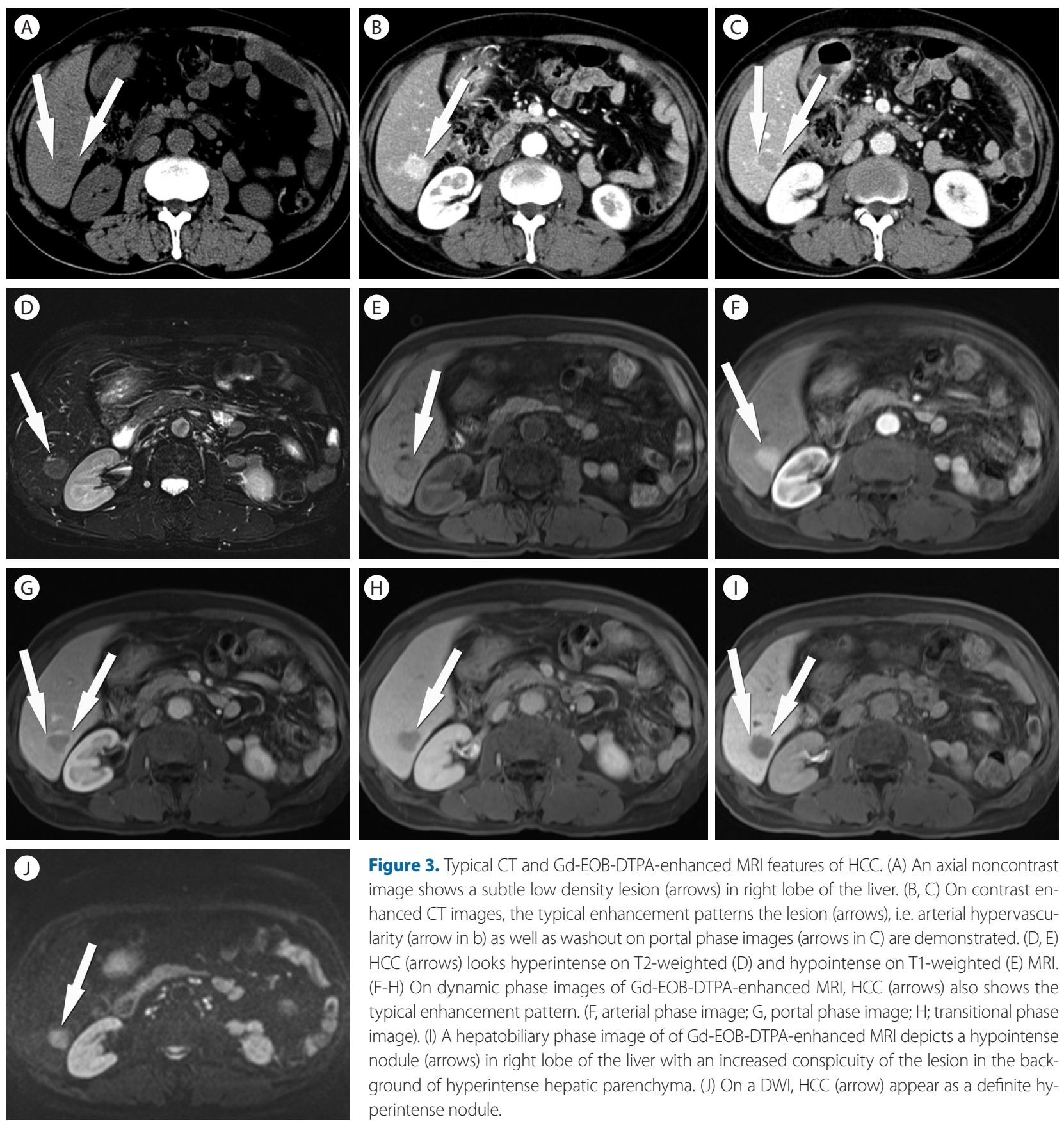

Figure 3. Typical CT and Gd-EOB-DTPA-enhanced MRI features of HCC. (A) An axial noncontrast image shows a subtle low density lesion (arrows) in right lobe of the liver. (B, C) On contrast enhanced $\mathrm{CT}$ images, the typical enhancement patterns the lesion (arrows), i.e. arterial hypervascularity (arrow in b) as well as washout on portal phase images (arrows in C) are demonstrated. (D, E) HCC (arrows) looks hyperintense on T2-weighted (D) and hypointense on T1-weighted (E) MRI. (F-H) On dynamic phase images of Gd-EOB-DTPA-enhanced MRI, HCC (arrows) also shows the typical enhancement pattern. $(F$, arterial phase image; $G$, portal phase image; $H$; transitional phase image). (I) A hepatobiliary phase image of of Gd-EOB-DTPA-enhanced MRI depicts a hypointense nodule (arrows) in right lobe of the liver with an increased conspicuity of the lesion in the background of hyperintense hepatic parenchyma. (J) On a DWI, HCC (arrow) appear as a definite hyperintense nodule.

supportive to increase the sensitivity for the diagnosis of HCCS with atypical vascular profiles (Fig. 4). ${ }^{5,6,35}$

Two recent significant advances in liver MRI are the introduction of hepatocyte-specific contrast agents such as Gd-EOB-DTPA and the application of DWI in routine clinical liver MR examination. Between the two currently available hepatocyte-specific contrast agents, Gd-EOB-DTPA is advantageous over Gd-BOPTA because the hepatocyte uptake percentage ( $50 \%$ of the administered) of
Gd-EOB-DTPA is greater than that (5\%) of Gd-BOPTA. As a result, the hepatobiliary phase imaging with Gd-EOB-DTPA yields superior hepatic enhancement and requires a time delay (20 minutes) shorter than that with Gd-BOPTA (60-120 minutes). ${ }^{36,37}$ In this hepatobiliary phase images, malignant tumors such as HCCs are spared from the contrast uptake that occurs in the surrounding liver because of the absent or the hampered function of hepatocytes in malignant tumors. ${ }^{17}$ 

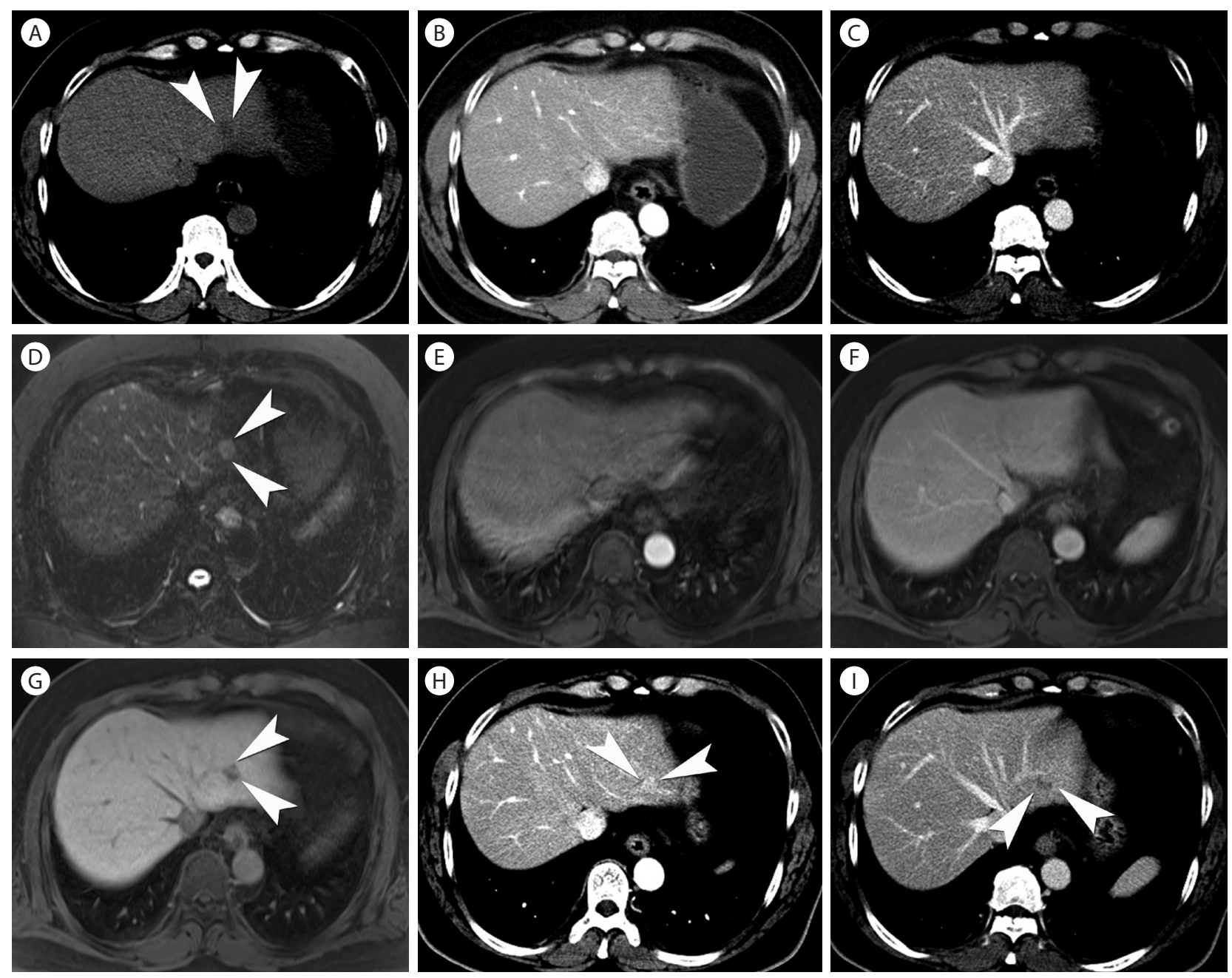

Figure 4. Atypical enhancement pattern of HCC. (A-C) In a male patient with chronic hepatitis B and an elevated level of alpha fetoprotein, the first contrast-enhanced CT fails to detect lesions suspicious of HCCs. On a noncontrast imge (A), a hypodense lesion (arrowheads) is found in left lobe of the liver, while it is not seen on arterial (B) and portal (C) phase images. (D-G) Gd-EOB-DTPA-enhanced MRI at the similar time of the first CT images, also fails to find typical vascular enhancement pattern of the lesion, although the lesion (arrowheads) is seen hyperintense on a T2-weighted image (D). A hepatobiliary phase image (G) demonstrates a hypointense lesion (arrowheads). (E, arterial phase image; F. portal phase image). (H,I) 6-month followup CT images finally define the typical arterial hypervascularity (arrowheads in $\mathrm{h}$ ) and washout on a portal phase image (arrowheads in I).

The assessment of added diagnostic value of the hepatobiliary phase imaging is currently in the field of active research. Several studies reported that Gd-EOB-DTPA enhanced MR outperformed CT for the diagnosis of HCC. ${ }^{38,39}$ In particular, Gd-EOB-DTPA was advantageous over $\mathrm{CT}$ for small lesions less than $1 \mathrm{~cm}^{40}$ and for the differentiation of small HCCs from hypervascular pseudolesions in patients with chronic liver disease. ${ }^{41,42}$ Moreover, Gd-EOBDTPA hepatobiliary phase imaging features can be used as biomarkers to predict microvascular invasion, tumor aggressiveness, and even patient's outcome. ${ }^{43-46}$

While Gd-EOB-DTPA-enhanced MRI offers a number of exciting opportunities as a new imaging tool, there remain some challeng- es in clinical application of Gd-EOB-DTPA-enhanced MRI. First, hypointense nodules that are observed only during the hepatobiliary phase are frequently encountered in routine clinical practice. Yet, the clinical significance of these nodules is unclear. Several studies reported that a considerable proportion (27.6-43.5\%) of non-specific hypointense nodules presented at the initial hepatobiliary phase images showed interval changes in their MR signal and morphological characteristics at follow-up MRI. ${ }^{47-50}$ Potential factors associated with the interval transformation of these lesions included the size ( $>15 \mathrm{~mm})$, tumor doubling time ( $<542$ days), the presence of fat, high signal intensity on T1-weighte images, and high signal intensity on DWI. ${ }^{47-50}$ The optimal follow-up interval 

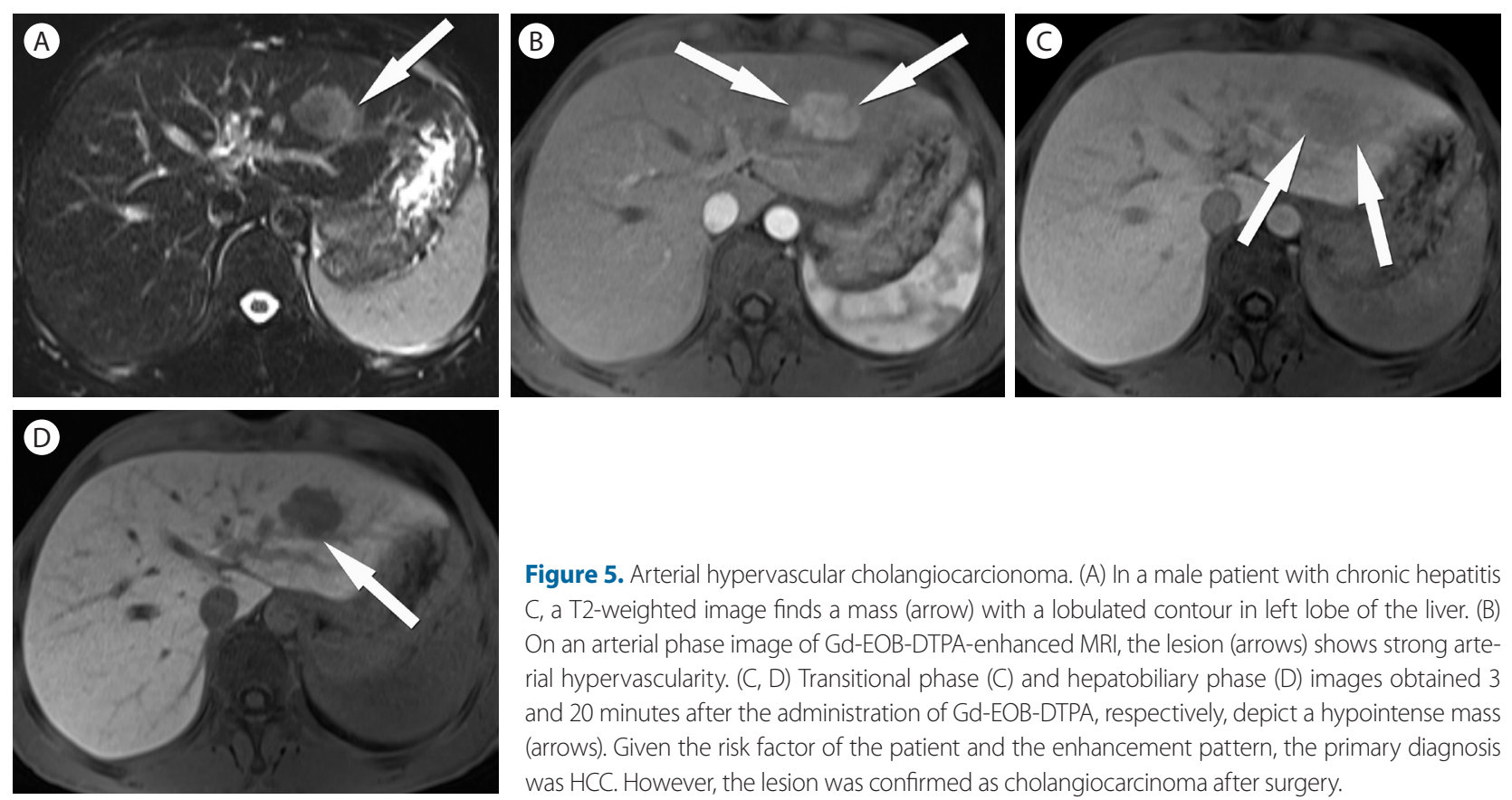

Figure 5. Arterial hypervascular cholangiocarcionoma. (A) In a male patient with chronic hepatitis C, a T2-weighted image finds a mass (arrow) with a lobulated contour in left lobe of the liver. (B) On an arterial phase image of Gd-EOB-DTPA-enhanced MRI, the lesion (arrows) shows strong arterial hypervascularity. (C, D) Transitional phase (C) and hepatobiliary phase (D) images obtained 3 and 20 minutes after the administration of Gd-EOB-DTPA, respectively, depict a hypointense mass (arrows). Given the risk factor of the patient and the enhancement pattern, the primary diagnosis was HCC. However, the lesion was confirmed as cholangiocarcinoma after surgery.

and treatment strategy for these hepatobiliary hypointense nodules are yet to be determined. Second, mass-forming intrahepatic cholangiocarciomas may mimic HCCs on Gd-EOB-DTPA-enhanced MRI (Fig. 5)..$^{51}$ Arterial hypervascularity was reported in $30 \%$ of intrahepatic cholangiocarcionomas, especially in small tumors less than $3 \mathrm{~cm} .{ }^{52}$ Furthermore, the characteristic delayed enhancement of cholangiocarcinoma could not be well demonstrated on $\mathrm{Gd}$ EOB-DTPA MRI because the inherently high background hepatocyte uptake may affect the imaging features on the delayed phase images contributed by the extracellular distribution of Gd-EOBDTPA contrast agent. ${ }^{51}$ Third, the acquisition of optimal arterial phase images in Gd-EOB-DTPA enhanced MR is more challenging than other conventional extracellular MRI contrast agent. ${ }^{53}$ This is likely due to the difference in the amount of administered contrast agents: Gd-EOB-DTPA typically administered at $0.1 \mathrm{~mL} / \mathrm{kg}$ body weight while conventional extracellular contrast agents at $0.2 \mathrm{~mL} /$ $\mathrm{kg}$ body weight. ${ }^{53}$ Weak arterial enhancement may negatively affect the conspicuity of detecting hypervascular lesions such as HCC. In addition, there was a report showing that transient tachypnea after administration of Gd-EOB-DTPA possibly decreased the quality of the arterial phase images of Gd-EOB-DTPA-enhanced $M{ }^{5}{ }^{54}$

DWI reflects the diffusion process of molecules, mainly water, in tissue and allows us to characterize tissue microstructural changes. A highly cellular tissue such as malignant tumor results in restriction of the apparent diffusion of water molecules and a de- crease in the apparent diffusion coefficient (ADC) values. ${ }^{17}$ As a result, liver tumors including HCCS appear as high signal intensity lesions on DWI in contrast to the low signal intensity of the liver parenchyma. ${ }^{55}$ Studies reported higher rates of detection of HCCs with added DWI (sensitivity of $84-98 \%$ ) than multiphasic MRI alone (sensitivity of $76-85 \%)^{5-58}$ although the results are still debatable. ${ }^{59}$ Combination of hepatobiliary phase images of Gd-EOB-DTPA and DWI would improve the diagnostic performance of MRI, especially for nodules with atypical vascular pattern and small size. ${ }^{60-62}$

\section{Guideline for imaging diagnosis of HCC}

The interpretation of liver imaging and the radiologic reporting become more complex with new, advanced imaging techniques. In a common clinical setting, patients often undergo multiple imaging studies that are interpreted by multiple radiologists. These added complexities lead to inconsistent interpretations and reporting of radiological studies. As an initiative to address this problem, the American College of Radiology proposed the Liver ImagingReporting and Data System (LI-RADS) (Accessed February 2015, from http://www.acr.org/Quality-Safety/Resources/LIRADS/). The goals of the initiative are to reduce variability in lesion interpretation by standardizing report content and structure; improving communication with clinicians; and facilitating decision making, outcome monitoring, performance auditing, quality assurance, and research. ${ }^{63}$ Five categories that follow the diagnostic thought pro- 
cess are used to stratify individual observations according to the level of concern for HCC. ${ }^{63}$ LI-RADS diagnostic algorithm is intended only for individuals at increased risk for HCC regardless of presence or absence of previous surveillance US or other imaging. Distinct from AASLD guideline, LI-RADS expanded the "Indeterminate" category into probably benign, intermediate probability of HCC and probably HCC (LI-RADS categories 2, 3 and 4) to minimize false positive interpretations for $1-2 \mathrm{~cm}$ nodule detected by $\mathrm{CT}$ or MRI. The latest LI-RADS 2014 includes considerations of cholangiocarcinoma, mixed hepatobiliary tumors, infiltrative HCC as well as the use of hepatobiliary MRI contrast agents. ${ }^{64}$

\section{CONCLUSION}

In summary, continued advances and changes are made in imaging studies for the detection and diagnosis of HCC. MRI with hepatocyte-specific contrast agents and DWI is increasingly accepted, in part because of their potentials for improved diagnosis of early HCCs. In comparison, SPIO-enhanced MRIs on decline because of limited availability of SPIO contrast agents and restricted clinical applicability. Double contrast MR and CT hepatic arteriorgraphy and arterioportography are less commonly used, given their demanding technical complexity. We should keep in mind that the current guidelines for the clinical applicability and appropriateness of imaging diagnosis of HCC, will be constantly refined and updated with on-going advances in imaging techniques and supportive data from clinical validation and research studies.

\section{Acknowledgements}

This study was supported by Grant \#2012R1A1A1012731 by Basic Science Research Program through the National Research Foundation of Korea (NRF) funded by the Ministry of Education, Science and Technology, Korea.

\section{Conflicts of Interest}

The authors have no conflicts to disclose.

\section{REFERENCES}

1. Theisis ND, Curado MP, Franceschi S, Hytiroglow P, Kudo M, Park YN, et al. Hepatocellular carcinoma. In: Bosman FT, Carneiro F, Hruban,
RH, Theise ND, ed. WHO Classification of Tumours of the Digestive System. Lyon, France: IARC Press, 2010:205-216.

2. Shariff MI, Cox IJ, Gomaa Al, Khan SA, Gedroyc W, Taylor-Robinson SD. Hepatocellular carcinoma: current trends in worldwide epidemiology, risk factors, diagnosis and therapeutics. Expert Rev Gastroenterol Hepatol 2009;3:353-367.

3. Llovet JM, Burroughs A, Bruix J. Hepatocellular carcinoma. Lancet 2003;362:1907-1917.

4. Bruix J, Sherman M, Llovet JM, Beaugrand M, Lencioni R, Burroughs AK, et al. Clinical management of hepatocellular carcinoma. Conclusions of the Barcelona-2000 EASL conference. European Association for the Study of the Liver. J Hepatol 2001;35:421-430.

5. Omata M, Lesmana LA, Tateishi R, Chen PJ, Lin SM, Yoshida H, et al. Asian Pacific Association for the Study of the Liver consensus recommendations on hepatocellular carcinoma. Hepatol Int 2010;4:439-474.

6. Bruix J, Sherman M, American Association for the Study of Liver D. Management of hepatocellular carcinoma: an update. Hepatology 2011;53:1020-1022.

7. Kudo M. Multistep human hepatocarcinogenesis: correlation of imaging with pathology. J Gastroenterol 2009;44 Suppl 19:112-118.

8. Hayashi M, Matsui O, Ueda K, Kawamori Y, Kadoya M, Yoshikawa J, et al. Correlation between the blood supply and grade of malignancy of hepatocellular nodules associated with liver cirrhosis: evaluation by CT during intraarterial injection of contrast medium. AJR Am J Roentgenol 1999;172:969-976.

9. Matsui O, Kadoya M, Kameyama T, Yoshikawa J, Takashima T, Nakanuma $Y$, et al. Benign and malignant nodules in cirrhotic livers: distinction based on blood supply. Radiology 1991;178:493-497.

10. Ueda K, Terada T, Nakanuma Y, Matsui O. Vascular supply in adenomatous hyperplasia of the liver and hepatocellular carcinoma: a morphometric study. Hum Pathol 1992;23:619-626.

11. Yanagisawa K, Moriyasu F, Miyahara T, Yuki M, lijima H. Phagocytosis of ultrasound contrast agent microbubbles by Kupffer cells. Ultrasound Med Biol 2007;33:318-325.

12. Gandhi SN, Brown MA, Wong JG, Aguirre DA, Sirlin CB. MR contrast agents for liver imaging: what, when, how. Radiographics 2006;26:1621-1636.

13. Kitao A, Matsui O, Yoneda N, Kozaka K, Shinmura R, Koda W, et al. The uptake transporter OATP8 expression decreases during multistep hepatocarcinogenesis: correlation with gadoxetic acid enhanced MR imaging. Eur Radiol 2011;21:2056-2066.

14. Colli A, Fraquelli M, Casazza G, Massironi S, Colucci A, Conte D, et al. Accuracy of ultrasonography, spiral CT, magnetic resonance, and alpha-fetoprotein in diagnosing hepatocellular carcinoma: a systematic review. Am J Gastroenterol 2006;101:513-523.

15. Lee MW, Kim YJ, Park HS, Yu NC, Jung SI, Ko SY, et al. Targeted sonography for small hepatocellular carcinoma discovered by CT or MRI: factors affecting sonographic detection. AJR Am J Roentgenol 
2010;194:W396-400.

16. Ayuso C, Rimola J, Garcia-Criado A. Imaging of HCC. Abdom Imaging 2012;37:215-230.

17. Lee JM, Yoon JH, Kim KW. Diagnosis of hepatocellular carcinoma: newer radiological tools. Semin Oncol 2012;39:399-409.

18. Yu SC, Yeung DT, So NM. Imaging features of hepatocellular carcinoma. Clin Radiol 2004;59:145-156.

19. Brannigan M, Burns PN, Wilson SR. Blood flow patterns in focal liver lesions at microbubble-enhanced US. Radiographics 2004;24:921935.

20. Kim TK, Lee KH, Khalili K, Jang HJ. Hepatocellular nodules in liver cirrhosis: contrast-enhanced ultrasound. Abdom Imaging 2011;36:244263.

21. Chen LD, Xu HX, Xie XY, Xie XH, Xu ZF, Liu GJ, et al. Intrahepatic cholangiocarcinoma and hepatocellular carcinoma: differential diagnosis with contrast-enhanced ultrasound. Eur Radiol 2010;20:743753.

22. Vilana R, Forner A, Bianchi L, Garcia-Criado A, Rimola J, de Lope $C R$, et al. Intrahepatic peripheral cholangiocarcinoma in cirrhosis patients may display a vascular pattern similar to hepatocellular carcinoma on contrast-enhanced ultrasound. Hepatology 2010;51:20202029.

23. Kudo M, Hatanaka K, Kumada T, Toyoda H, Tada T. Double-contrast ultrasound: a novel surveillance tool for hepatocellular carcinoma. Am J Gastroenterol 2011;106:368-370.

24. Nicolau C, Catala V, Vilana R, Gilabert R, Bianchi L, Sole M, et al. Evaluation of hepatocellular carcinoma using SonoVue, a second generation ultrasound contrast agent: correlation with cellular differentiation. Eur Radiol 2004;14:1092-1099.

25. Sangiovanni A, Manini MA, lavarone M, Romeo R, Forzenigo LV, Fraquelli $\mathrm{M}$, et al. The diagnostic and economic impact of contrast imaging techniques in the diagnosis of small hepatocellular carcinoma in cirrhosis. Gut 2010;59:638-644.

26. Khalili K, Kim TK, Jang HJ, Haider MA, Khan L, Guindi M, et al. Optimization of imaging diagnosis of $1-2 \mathrm{~cm}$ hepatocellular carcinoma: an analysis of diagnostic performance and resource utilization. J Hepatol 2011;54:723-728.

27. Burrel M, Llovet JM, Ayuso C, Iglesias C, Sala M, Miquel $R$, et al. MRI angiography is superior to helical CT for detection of HCC prior to liver transplantation: an explant correlation. Hepatology 2003;38:1034-1042.

28. de Ledinghen $V$, Laharie $D$, Lecesne $R$, Le Bail B, Winnock M, Bernard $\mathrm{PH}$, et al. Detection of nodules in liver cirrhosis: spiral computed tomography or magnetic resonance imaging? A prospective study of 88 nodules in 34 patients. Eur J Gastroenterol Hepatol 2002;14:159-165.

29. Tomemori T, Yamakado K, Nakatsuka A, Sakuma H, Matsumura K, Takeda K. Fast 3D dynamic MR imaging of the liver with MR SmartPrep: comparison with helical CT in detecting hypervascular hepato- cellular carcinoma. Clin Imaging 2001;25:355-361.

30. Rode A, Bancel B, Douek P, Chevallier M, Vilgrain V, Picaud G, et al. Small nodule detection in cirrhotic livers: evaluation with US, spiral CT, and MRI and correlation with pathologic examination of explanted liver. J Comput Assist Tomogr 2001;25:327-336.

31. Yoon SH, Lee JM, So YH, Hong SH, Kim SJ, Han JK, et al. Multiphasic MDCT enhancement pattern of hepatocellular carcinoma smaller than $3 \mathrm{~cm}$ in diameter: tumor size and cellular differentiation. AJR Am J Roentgenol 2009;193:W482-489.

32. Bolondi L, Gaiani S, Celli N, Golfieri R, Grigioni WF, Leoni S, et al. Characterization of small nodules in cirrhosis by assessment of vascularity: the problem of hypovascular hepatocellular carcinoma. Hepatology 2005;42:27-34.

33. Kojiro M, Roskams T. Early hepatocellular carcinoma and dysplastic nodules. Semin Liver Dis 2005;25:133-142.

34. Sherman M. The radiological diagnosis of hepatocellular carcinoma. Am J Gastroenterol 2010;105:610-612.

35. Tan CH, Low SC, Thng CH. APASL and AASLD Consensus Guidelines on Imaging Diagnosis of Hepatocellular Carcinoma: A Review. Int J Hepatol 2011;2011:519783.

36. Filippone A, Blakeborough A, Breuer J, Grazioli L, Gschwend S, Hammerstingl $R$, et al. Enhancement of liver parenchyma after injection of hepatocyte-specific MRI contrast media: a comparison of gadoxetic acid and gadobenate dimeglumine. J Magn Reson Imaging 2010;31:356-364.

37. Park G, Kim YK, Kim CS, Yu HC, Hwang SB. Diagnostic efficacy of gadoxetic acid-enhanced MRI in the detection of hepatocellular carcinomas: comparison with gadopentetate dimeglumine. $\mathrm{Br} J$ Radiol 2010;83:1010-1016.

38. Di Martino M, Marin D, Guerrisi A, Baski M, Galati F, Rossi M, et al. Intraindividual comparison of gadoxetate disodium-enhanced MR imaging and 64-section multidetector $C T$ in the Detection of hepatocellular carcinoma in patients with cirrhosis. Radiology 2010;256:806-816.

39. Akai H, Kiryu S, Matsuda I, Satou J, Takao H, Tajima T, et al. Detection of hepatocellular carcinoma by Gd-EOB-DTPA-enhanced liver MRI: comparison with triple phase 64 detector row helical CT. Eur J Radiol 2011;80:310-315.

40. Kim SH, Kim SH, Lee J, Kim MJ, Jeon YH, Park Y, et al. Gadoxetic acid-enhanced MRI versus triple-phase MDCT for the preoperative detection of hepatocellular carcinoma. AJR Am J Roentgenol 2009;192:1675-1681.

41. Motosugi U, Ichikawa T, Sou H, Sano K, Tominaga L, Muhi A, et al. Distinguishing hypervascular pseudolesions of the liver from hypervascular hepatocellular carcinomas with gadoxetic acid-enhanced MR imaging. Radiology 2010;256:151-158.

42. Sun HY, Lee JM, Shin Cl, Lee DH, Moon SK, Kim KW, et al. Gadoxetic acid-enhanced magnetic resonance imaging for differentiating small 
hepatocellular carcinomas ( $<$ or $=2 \mathrm{~cm}$ in diameter) from arterial enhancing pseudolesions: special emphasis on hepatobiliary phase imaging. Invest Radiol 2010;45:96-103.

43. Kim KA, Kim MJ, Jeon HM, Kim KS, Choi JS, Ahn SH, et al. Prediction of microvascular invasion of hepatocellular carcinoma: usefulness of peritumoral hypointensity seen on gadoxetate disodium-enhanced hepatobiliary phase images. J Magn Reson Imaging 2012;35:629634.

44. Kim JY, Kim MJ, Kim KA, Jeong HT, Park YN. Hyperintense HCC on hepatobiliary phase images of gadoxetic acid-enhanced MRI: correlation with clinical and pathological features. Eur J Radiol 2012:81:3877-3882.

45. Kitao A, Matsui O, Yoneda N, Kozaka K, Kobayashi S, Koda W, et al. Hypervascular hepatocellular carcinoma: correlation between biologic features and signal intensity on gadoxetic acid-enhanced MR images. Radiology 2012;265:780-789.

46. Choi JW, Lee JM, Kim SJ, Yoon JH, Baek JH, Han JK, et al. Hepatocellular carcinoma: imaging patterns on gadoxetic acid-enhanced MR Images and their value as an imaging biomarker. Radiology 2013;267:776-786.

47. Higaki A, Ito K, Tamada T, Teruki S, Yamamoto A, Higashi H, et al. High-risk nodules detected in the hepatobiliary phase of $\mathrm{Gd}$ EOB-DTPA-enhanced mr imaging in cirrhosis or chronic hepatitis: Incidence and predictive factors for hypervascular transformation, preliminary results. J Magn Reson Imaging 2013;37;1377-1383.

48. Kumada T, Toyoda H, Tada T, Sone Y, Fujimori M, Ogawa S, et al. Evolution of hypointense hepatocellular nodules observed only in the hepatobiliary phase of gadoxetate disodium-enhanced MRI. AJR Am J Roentgenol 2011;197:58-63.

49. Hyodo T, Murakami T, Imai Y, Okada M, Hori M, Kagawa Y, et al. Hypovascular nodules in patients with chronic liver disease: risk factors for development of hypervascular hepatocellular carcinoma. Radiology 2013;266:480-490.

50. Kim YK, Lee WJ, Park MJ, Kim SH, Rhim H, Choi D. Hypovascular hypointense nodules on hepatobiliary phase gadoxetic acidenhanced MR images in patients with cirrhosis: potential of DW imaging in predicting progression to hypervascular HCC. Radiology 2012;265:104-114.

51. Chong YS, Kim YK, Lee MW, Kim SH, Lee WJ, Rhim HC, et al. Differentiating mass-forming intrahepatic cholangiocarcinoma from atypical hepatocellular carcinoma using gadoxetic acid-enhanced MRI. Clin Radiol 2012;67:766-773.

52. Kim SA, Lee JM, Lee KB, Kim SH, Yoon SH, Han JK, et al. Intrahepatic mass-forming cholangiocarcinomas: enhancement patterns at multiphasic CT, with special emphasis on arterial enhancement pattern-correlation with clinicopathologic findings. Radiology 2011;260:148157.

53. Ringe KI, Husarik DB, Sirlin CB, Merkle EM. Gadoxetate disodium- enhanced MRI of the liver: part 1, protocol optimization and lesion appearance in the noncirrhotic liver. AJR Am J Roentgenol 2010;195:13-28.

54. Davenport MS, Viglianti BL, Al-Hawary MM, Caoili EM, Kaza RK, Liu PS, et al. Comparison of acute transient dyspnea after intravenous administration of gadoxetate disodium and gadobenate dimeglumine: effect on arterial phase image quality. Radiology 2013;266:452-461.

55. Bolog N, Andreisek G, Oancea I, Mangrau A. CT and MR imaging of hepatocellular carcinoma. J Gastrointestin Liver Dis 2011;20:181-189.

56. Vandecaveye V, De Keyzer F, Verslype C, Op de Beeck K, Komuta M, Topal $B$, et al. Diffusion-weighted MRI provides additional value to conventional dynamic contrast-enhanced MRI for detection of hepatocellular carcinoma. Eur Radiol 2009;19:2456-2466.

57. Xu PJ, Yan FH, Wang JH, Lin J, Ji Y. Added value of breathhold diffusion-weighted MRI in detection of small hepatocellular carcinoma lesions compared with dynamic contrast-enhanced MRI alone using receiver operating characteristic curve analysis. J Magn Reson Imaging 2009;29:341-349.

58. Piana G, Trinquart L, Meskine N, Barrau V, Beers BV, Vilgrain V. New MR imaging criteria with a diffusion-weighted sequence for the diagnosis of hepatocellular carcinoma in chronic liver diseases. J Hepatol 2011;55:126-132.

59. Park MS, Kim S, Patel J, Hajdu CH, Do RK, Mannelli L, et al. Hepatocellular carcinoma: detection with diffusion-weighted versus contrast-enhanced magnetic resonance imaging in pretransplant patients. Hepatology 2012;56:140-148.

60. Lee MH, Kim SH, Park MJ, Park CK, Rhim H. Gadoxetic acidenhanced hepatobiliary phase MRI and high-b-value diffusionweighted imaging to distinguish well-differentiated hepatocellular carcinomas from benign nodules in patients with chronic liver disease. AJR Am J Roentgenol 2011;197:W868-875.

61. Park MJ, Kim YK, Lee MW, Lee WJ, Kim YS, Kim SH, et al. Small hepatocellular carcinomas: improved sensitivity by combining gadoxetic acid-enhanced and diffusion-weighted MR imaging patterns. Radiology 2012;264:761-770.

62. Park MJ, Kim YK, Lee MH, Lee JH. Validation of diagnostic criteria using gadoxetic acid-enhanced and diffusion-weighted MR imaging for small hepatocellular carcinoma $(<=2.0 \mathrm{~cm})$ in patients with hepatitis-induced liver cirrhosis. Acta Radiol 2013;54:127-136.

63. Purysko AS, Remer EM, Coppa CP, Leao Filho HM, Thupili CR, Veniero JC. LI-RADS: a case-based review of the new categorization of liver findings in patients with end-stage liver disease. Radiographics 2012;32:1977-1995.

64. Mitchell DG, Bruix J, Sherman M, Sirlin CB. LI-RADS (Liver Imaging Reporting and Data System): Summary, discussion, consensus of the LI-RADS Management Working Group and future directions. Hepatology 2015;61:1056-1065. 